\title{
Chinese Character Teaching and Countermeasures in Teaching Chinese as a Foreign Language
}

\author{
ShuanJun $\mathrm{An}^{1, *}$, WeiHua $\mathrm{Hu}^{2, \mathrm{a}}$

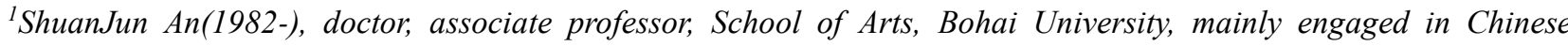 \\ phonology and Chinese dialectology. \\ ${ }^{2}$ WeiHua Hu(1996-), studying for a master's degree, School of Arts, Bohai University, mainly engaged in the research \\ of teaching Chinese to speakers of other language. \\ *E-mail: anshuanjun123@126.com,E-mail:1826516216@qq.com
}

\begin{abstract}
Chinese characters are different from other characters in the structure, and they have become symbols representing the Chinese nation in the long process of development. This requires teachers of Chinese as a foreign language in teaching start from practice, from the characteristics of Chinese characters, recognizing the problems in Chinese character teaching, and to continuously explore and improve on the basis of the lessons of predecessors, so that Chinese character teaching can play a greater role. Starting from the current situation of Chinese character teaching, the author conducts a comprehensive analysis and puts forward reasonable countermeasures in combination with the problems of Chinese character teaching in Bohai University's teaching of Chinese as a foreign language.
\end{abstract}

Keywords : Teaching Chinese as a foreign language, Chinese character teaching, Countermeasures

\section{INTRODUCTION}

As a special written symbol system for recording Chinese, Chinese characters occupy an important position in teaching Chinese as a foreign language and have unique values. Both the acquisition of the first language and the learning of the second language must start with Chinese characters, and the teaching of pronunciation, vocabulary and grammar is often combined with the teaching of Chinese characters. In recent years, the teaching of Chinese characters has received increasing attention in teaching Chinese as a foreign language. As the carrier of Chinese culture, Chinese characters can be understood more smoothly by mastering Chinese characters, which can help learners learn and use Chinese better. For foreign students, they do not have a Chinese learning environment, and Chinese learning is the study and use of Chinese characters. At present, Chinese character teaching has been paid more and more attention in teaching Chinese as a foreign language, but there are still some problems. Since the teaching of Chinese characters is very important, and there are still some problems in the teaching of Chinese characters, the teaching of Chinese characters has been chosen as the subject of research.
As a special written symbol system for recording Chinese, Chinese characters occupy an important position in teaching Chinese as a foreign language and have unique values. In recent years, the teaching of Chinese characters has received increasing attention in teaching Chinese as a foreign language.

\section{AN OVERVIEW OF THE TEACHING OF CHINESE CHARACTERS IN TEACHING CHINESE AS A FOREIGN LANGUAGE}

In recent years, China's comprehensive national strength has gradually improved. As a result, Chinese has received widespread attention from people from all walks of life around the world. The international status of Chinese has gradually increased. The learning of Chinese is the general trend, and the "Chinese fever" is sweeping the world.

\subsection{The Importance of Chinese Character Teaching in Teaching Chinese as a Foreign Language}

From the perspective of Chinese characters, as one of the important carriers of Chinese culture, Chinese characters carry profound Chinese cultural heritage. 
From the oracle bone inscriptions in the Yin and Shang dynasties to the current Chinese characters, to a certain extent, it can be said that Chinese characters have witnessed the growth and development of Chinese culture. In the teaching of Chinese as a foreign language, the teaching of Chinese characters can greatly enhance students' interest in learning Chinese, at the same time, deepen the understanding of Chinese characters and the culture behind them. Chinese characters belong to the ideographic system. The shape of Chinese characters can express a certain meaning. "A syllable in Chinese is not recorded by one Chinese character. There are more than 400 Chinese syllables, but it is represented by tens of thousands of characters." [1] As a "stepping stone" in the teaching of Chinese as a foreign language, Chinese characters occupy a large part of the teaching of various elements of Chinese as a foreign language.

\subsection{The purpose and thinking of Chinese character teaching in teaching Chinese as a foreign language}

"The teaching of Chinese characters is a complete process of perception and memory of form, sound, and meaning. In this process, stroke teaching, component teaching, stroke order teaching and characters are taught from words."[2] Stroke is the smallest unit that constitutes Chinese characters. In the initial stage of Chinese character teaching, you generally start teaching from strokes, and then begin to familiarize yourself with stroke order in the next phase. After mastering the strokes and stroke order, you will enter the recognition stage, and finally spell the entire Chinese character and teach the characters from the words. Following the teaching sequence from part to whole can reduce students' fear of difficulty and enhance their confidence in learning.

\subsection{The important and difficult points of Chinese character teaching in teaching Chinese as a foreign language}

The teaching of Chinese characters is a key step in laying a good foundation in teaching Chinese as a foreign language. It is the cornerstone. If you want to build a high-rise building, you must fight the first battle of teaching Chinese characters. Stroke and stroke order are also the key part of this. If students do not give stroke order to students when teaching Chinese characters, learners will not be able to start when they write Chinese characters.

The problem of "difficulty of Chinese characters" is considered to be the most difficult problem in Chinese language learning. In the teaching of Chinese as a foreign language, there is a certain degree of difficulty in teaching Chinese characters, which is mainly determined by the characteristics of Chinese characters.
Chinese characters, as an ideographic system, do not have lengths and shorts and are arranged linearly like pinyin characters. No matter if there are many strokes or few strokes in Chinese characters, all the strokes are written in the same square, forming different glyphs criss-crossed in a square, the structure is complex, and the strokes are many, as many as 20 or 30 strokes. Stroke order is diverse and difficult to remember; there are many homophonic and polyphonic characters, which are easy to make mistakes, and it is difficult to fully grasp.

\section{PROBLEMS IN THE TEACHING OF CHINESE CHARACTERS IN TEACHING CHINESE AS A FOREIGN LANGUAGE}

\subsection{Relatively few teaching hours of Chinese characters}

In the teaching of Chinese as a foreign language, most of the time, the teaching of Chinese characters is only regarded as part of the vocabulary teaching. The teaching of Chinese characters is mixed in the vocabulary teaching, and there is no special Chinese character course, which causes the effect of Chinese character teaching to be greatly reduced. The teaching of Chinese characters has received the attention of the teaching of Chinese as a foreign language after the 5th International Chinese Teaching Conference was held. However, the teaching of Chinese characters is still a weak link in the overall view. In Bohai University's language students' classes, there is no special handwriting class. There are only a small part of the comprehensive class that teaches handwriting. In the elementary reading and writing class, handwriting is not fully taught; and there is a course for the degree students. Writing class only two class hours a week which is 36 class hours after a semester.

\subsection{Lack of flexibility in teaching methods}

The traditional teaching method of Chinese as a foreign language is single and lacks innovation. It blindly adheres to the method and mechanism of teacher writing, student learning, teacher demonstration, and student imitating. The entire classroom learning atmosphere is boring and students are not interested, and even tired of Chinese characters and Chinese classes.

\subsection{Errors in Chinese Characters Existed by Foreign Students}

Students from different countries and regions have different mother tongues and different levels of understanding of the target language. Due to the influence of many factors, students will have errors in Chinese characters. For example, European and American students who use pinyin as their mother 
tongue do not have homophones in English. Words and polyphonic characters will cause errors in this aspect when learning Chinese characters.

It is found from the classrooms of foreign students at the elementary level of Bohai University that the students at the elementary level do not have a deep grasp of Chinese characters. They often imitate Chinese characters from teachers or textbooks. In the process of imitation, it is easy to make mistakes such as replacement or deformation of parts. For example, when learning the two characters "lang, lang", the parts on the right half will be changed, causing confusion between these two characters. Similarly, taking "lang" as an example, some foreign students will deform " $f u$ " into "jie" when writing; mistakes in strokes also occur from time to time. When learning the "clean" word for "gan", more than half of them African students write it as "thousands".

\subsection{Unreasonable Chinese character teaching materials}

Chinese character teaching materials lack systemicity. The emphasis on Chinese character teaching has always been low, resulting in a relatively lack of Chinese character teaching materials, and the content and format design of some teaching materials is not reasonable enough, and the content of many teaching materials is partial rather than comprehensive. "Some focus on the teaching method of Chinese character radicals, and some focus on the teaching method of pictures, and lack a more comprehensive and systematic teaching material." [3] The difficulty level of the textbook is unreasonable. The difficulty setting of Chinese characters in some textbooks is unreasonable. There have been complicated and difficult Chinese characters in the elementary textbooks, which has increased the difficulty for teachers and learners. In the intermediate and advanced textbooks, there are some too simple Chinese characters, which students learn very well. Easy, reducing the mystery of Chinese characters.

\subsection{Lack of an effective connection between inside and outside the class}

In the teaching of Chinese characters for foreign students, generally, the knowledge learned in class is rarely brought into the class. More often, the teacher assigns some homework at the end of the class, requiring students to complete, and using compulsory teaching methods to make students as hard as possible. Knowledge learned in class. The connection of knowledge inside and outside the class has not been handled well. If the knowledge learned in class is not used outside the class, it is equivalent to talking on paper, with empty theory and no practice.

\section{THE CAUSES OF THE PROBLEMS IN THE TEACHING OF CHINESE CHARACTERS IN THE TEACHING OF CHINESE AS A FOREIGN LANGUAGE}

\subsection{The characteristics of Chinese characters and the over-generalization of target language knowledge}

Chinese characters are ideographic characters with a long history, representing the culture of China for 5000 years, and the number is huge. As one of the most difficult points in second language teaching, there will be great difficulties in recognition, reading, writing, etc. It is difficult for learners to learn, and it is difficult for teachers to teach.

When studying Chinese characters, foreign students will improperly apply the limited and insufficient knowledge of Chinese characters that they have learned to new Chinese character phenomena, causing errors in Chinese characters. For example, take South Korean students from Bohai University as an example. After they study "men", they will use the word "men" in the correct group of words such as "people" and "we", but they will also generalize them incorrectly. Used for the group of words in "big men", to confuse it with the homophone of "men".

\subsection{Individual differences of learners and negative transfer of mother tongue}

In the class of teaching Chinese as a foreign language, learners come from different countries and regions, with different goals to learn Chinese, and the difficulty requirements and mastery of Chinese characters are different. Some learners are interested in the Chinese culture carried by Chinese characters, and learn Chinese characters with curiosity about Chinese culture, so as to satisfy their understanding of Chinese culture; some people are because of the needs of work.

Foreign students from the non-Chinese character circle are different from native Chinese learners. They have formed a set of native language habit before learning Chinese characters. When learning Chinese characters, they are often easily affected by their mother tongue, which is the problem of mother tongue transfer.

\section{COUNTERMEASURES OF CHINESE CHARACTER TEACHING IN TEACHING CHINESE AS A FOREIGN LANGUAGE}

"In the teaching of Chinese characters, we must change our thinking and fully realize the special status and basic role of Chinese character teaching in the teaching of Chinese as a foreign language. As the intersection of pronunciation, vocabulary, and grammar, 
Chinese characters are the focus of teaching Chinese as a foreign language, not a vassal." [4] In the preparation for teaching Chinese characters as a foreign language and in the process of teaching Chinese characters as a foreign language, the correct medicine is prescribed to ensure that the teaching of Chinese characters for teachers of Chinese as a foreign language and the learners are closely integrated. Mainly can be improved from the following aspects:

\subsection{Improve the teaching methods of Chinese characters}

In teaching, we should flexibly use a variety of teaching methods, pay attention to teaching students in accordance with their aptitude, comprehensively consider multiple factors, and pay attention to the age of the teaching object, the level of learning, and the degree of mastery of Chinese characters required. Teachers should innovate in the traditional teaching methods of reading, comprehension, spelling, and application. For example, in the reading and writing class of foreign students, we can combine writing Chinese characters with paper-cutting, drawing Chinese characters on the paper-cutting, and then cutting out the Chinese characters according to the outline. This new teaching method enriches the hands-on ability of foreign students, and the most important thing is combines Chinese characters and traditional Chinese talent paper-cutting together, allowing students to appreciate the charm of Chinese characters and paper-cutting while learning Chinese characters, and enhancing learners' confidence and interest in Chinese character learning.

\subsection{Combination of theory and practice}

When teaching the basic strokes, stroke order, components and some representative Chinese characters of Chinese characters, teachers can combine cultural knowledge related to Chinese characters. For example, when teaching the Chinese character "nian", you can briefly introduce what is related to the culture of "nian", for example, why is it called the New Year? What is the relationship between folk's keeping of the year and the year? While achieving good results, it can also increase learners' interest and continuous motivation for Chinese character learning. In the classrooms of Bohai University, which are mainly composed of Korean and African students, teachers pay great attention to the extension of Chinese characters-related cultural knowledge in the classroom. When talking about Chinese characters, they mentioned the historical story of Cang jie characters. Teachers considered that students' level of Chinese characters also enriches the content of Chinese characters.

\subsection{Make full use of modern educational technology and resources}

With the continuous development of society, multimedia technology has also continued to advance and has been widely used in various fields. Modern educational technology has also been used in teaching Chinese as a foreign language. In fact, the use of modern educational technology is the general trend, and Chinese character teaching also needs it to bloom, greater brilliance. In the teaching of Chinese characters, multimedia can be used in many cases to promote better teaching effects. For example, when teaching strokes and stroke order, multimedia is used to dynamically display the process of stroke direction and stroke order. Learners seem to be clear at a glance, which can be better and faster Mastery. In addition to the use of multimedia resources in class, teachers can also use a combination of in-class and extra-curricular resources. For example, international students studying at Bohai University have WeChat APP. Teachers can seize this resource and establish a WeChat public account related to Chinese characters. Usually publish some interesting Chinese characters on the public account, historical stories related to Chinese characters, and so on.

\subsection{Rationalization of the selection of Chinese character teaching materials}

Textbooks are the blueprint for the class, and their systematic and scientific nature is related to the teaching effect. Therefore, the selection of Chinese character textbooks should be paid attention to. Different types of textbooks should be selected reasonably, and the knowledge of Chinese characters should be arranged in stages and levels. For example, The difficulty level of the textbooks at different stages should be appropriate and reasonable, and at the same time distinguishable, avoiding mechanically repeated and exercises the content of textbook, increasing summary exercises and targeted reading materials, so that Chinese characters appear in meaningful language forms, and learners can improve their cognitive level and learning effect.

\section{CONCLUSION}

The teaching of Chinese characters is an important part of teaching Chinese as a foreign language. With the continuous improvement of the status of Chinese in the world, the number of learners continue to increase. Chinese teaching activities have also achieved certain practical results, which means that the teaching of Chinese characters has also been advanced. In the teaching of Chinese characters, it is necessary to inherit the excellent teaching methods and forms of the predecessors, and continue to innovate and optimize. Every step of the teaching of Chinese characters must be strictly grasped and not slack. While disseminating the 
theoretical knowledge of Chinese characters, we should also pay attention to practice and integrate Chinese character teaching with special activities, such as calligraphy, Chinese painting, music, and paper-cutting to actively spread Chinese culture. In short, we must be down-to-earth, on the basis of absorbing previous experience, find the current deficiencies in Chinese character teaching, and continue to move forward. While continuously improving the problems in the teaching of Chinese characters, the teaching of Chinese characters should be placed in an important position, and the further research should be carried out to make Chinese characters play a greater role in the teaching of Chinese as a foreign language.

\section{Fund Project}

The key projects of social science planning fund of Liaoning Province "Investigation and research on dialects in the border area between Liaoning, Hebei and Inner Mongolia from the perspective of geolinguistics" (Project No: L19AYY005)

\section{REFERENCES}

[1] Huang Borong, Liao Xudong, Chinese in the Han Dynasty (fifth revised edition) Volume $1[\mathrm{M}]$, Beijing: Higher Education Press, 2011, 6.

[2] Wan Kaiyan, The use of phonetic symbols of pictophonetic characters in teaching Chinese as a foreign language $[\mathrm{J}]$, Science and Education Wenhui, 2018 (12).

[3] Xu Rui, Research on Teaching Strategies of Chinese as a Foreign Language [J], Modern Chinese (Academic Comprehensive Edition), 2016(07):156-157.

[4] Zheng Lanxin, The Theory of Characters as the Standard and Its Application in Teaching Chinese as a Foreign Language [J], Modern Chinese, 2018(02):182-186. 